CORRESPONDENCE

\title{
Early free light chain reduction following treatment initiation predicts favorable outcome in intact immunoglobulin myeloma
}

(c) The Author(s) 2021

Blood Cancer Journal (2022)12:3 ; https://doi.org/

10.1038/s41408-021-00600-6

\section{Dear Editor,}

Multiple myeloma (MM) is characterized by the proliferation of malignant plasma cells [1]. The neoplastic clones usually secrete an intact immunoglobulin (ilg) or fragments of immunoglobulin (lg) such as light chains. Intact immunoglobulins are traditionally measured by serum (SPEP) and urine protein electrophoresis, which are currently the gold standards to assess MM [2]. However, due to long half-lives of ilgs, SPEP is limited in its capacity to detect early changes after initiation of treatment.

MM displays enormous clonal heterogeneity with myeloma cell clones secreting ilg while other clones secreting the corresponding free light chain (FLC). These serum FLCs (sFLCs) have a short half-life of a few hours depending on renal function. The role of sFLCs as a prognostic factor at baseline and in the assessment of stringent complete response $(\mathrm{sCR})$ is well established $[3,4]$. In addition, Ig heavy/light chain analysis (HLC) combines measurement of individual Ig heavy chain isotypes with either $\mathrm{k}$ or $\lambda$ light chains [5]. The analysis allows accurate quantification of the involved and uninvolved lgs and permits to obtain a ratio of monoclonal and polyclonal lgs of the patient's affected isotype.

The primary aim of this study was to investigate the clinical value of serial SFLC and HLC measurements during the early phase of treatment, compare them to standard serum M-protein quantification by SPEP and evaluate the impact on progressionfree survival (PFS).

Between 2012 and 2014, consecutive newly diagnosed and relapsed MM patients with measurable disease by ilg $(\geq 10 \mathrm{~g} / \mathrm{L})$ and SFLC $(\geq 100 \mathrm{mg} / \mathrm{L})$ were prospectively identified [6]. Patients on dialysis were excluded. Informed consent was obtained in all patients in accordance with the Declaration of Helsinki

Serum samples for quantitative measurement of the paraprotein were obtained at initiation of treatment and thereafter every week for the first 3 cycles. The duration of each cycle was 3 or 4 weeks depending on the treatment regimens. SPEP was performed on Hydrasis (Sebia,) and sFLC with the Freelite assay (The Binding Site Inc) on an Immage nephelometer (Beckman Coulter). HLCs were measured using the Hevylite ${ }^{\circledR}$ assay (The binding Site Inc) on the BN II (Siemens Healthineers).

Responses for each paraprotein assay were descriptive and based on $\%$ of reduction compared to baseline: $\geq 90 \%$ reduction versus $\geq 50 \%$ reduction versus $<50 \%$ reduction. The unstable response was defined as an unsustained decrease in sFLC by $\geq 25 \%$ followed by an increase by $\geq 25 \%$ and $\geq 25 \mathrm{mg} / \mathrm{L}$ within the same cycle. A $90 \%$ cutoff for involved sFLC was used to make easier the comparison with the $90 \%$ cutoff for serum M-protein as per IMWG uniform response criteria [6].
Multivariate logistic regression was used to assess the association between best paraprotein response by SPEP and achieving $\geq 50 \%$ of paraprotein reduction after cycle 1 by sFLC. The probabilities of PFS were estimated using the Kaplan-Meier method. The designated time point for landmark analysis was the end of cycle 1 and the end of cycle 3. Regression analysis using Cox proportional hazard regression models was used to determine factors influencing outcomes. Statistical analyses were conducted using SAS (SAS Institute Inc).

We evaluated 30 episodes of treatment among 24 patients. The median age was 63 years (range: $39-89$ ). Per eligibility criteria, all MM episodes studied secreted an ilg: $23 \mathrm{lgG}$ and $7 \mathrm{lgA}$. Treatment regimens were bortezomib-based in $57 \%$, lenalidomide-based in $23 \%$ or pomalidomide-based in $17 \%$ of patients. Seven episodes of treatment were in newly diagnosed MM patients, the remaining episodes in relapsed disease (median of 4 prior lines of treatment; range $2-8$ ). Patients received an autologous stem cell transplant in 12 episodes of treatment and an allogeneic transplant in one. In addition, 11 episodes (37\%) were in patients participating in clinical trials receiving experimental agents in combination with a bortezomib- or pomalidomide-based treatment. The median follow-up was 85 months. By the end of cycle $1, a \geq 50 \%$ reduction was achieved in $20 \%$ by SPEP, $53 \%$ by sFLC, and $27 \%$ by HLC. This reduction by SFLC preceded the one by SPEP by a median of 3 weeks and the one by HLC by a median of 2.5 weeks.

In addition, 7 of 11 (64\%) treatment episodes who did not achieve $\geq 50 \%$ paraprotein reduction by SPEP did not achieve $\geq 50 \%$ of reduction by FLC. Response by SFLC at the end of cycle 1 correlated with the best response achieved by SPEP on treatment, while these observations were not seen with HLCs.

Patients with paraprotein reduction measured by sFLC $\geq 90 \%$ by the end of cycle 1 had a better 1-year PFS, compared to those with $<90 \%$ reduction $(63 \%$ vs. $36 \%, p=0.047$; Fig. $1 \mathrm{~A})$. After multivariate adjustment for ISS (HR 1.09, $p=0.883$ ), reduction of paraprotein $\geq 50 \%$ by SPEP after cycle 1 (HR $=1.79, p=0.427)$, only reduction of paraprotein $\geq 90 \%$ by sFLC after cycle 1 (HR 0.23 , $p=0.011$ ) remained predictive of better PFS.

Similarly, among patients with $<50 \%$ paraprotein reduction by SPEP at the end of cycle 1 , those having a $\geq 90 \%$ paraprotein reduction by sFLC showed a longer 1-year PFS compared to those with less than $90 \%$ reduction $(100 \%$ vs. $35 \%, p=0.049)$. In the multivariate logistic regression, achieving $\geq 50 \%$ of paraprotein reduction by sFLC after cycle 1 was strongly associated with a late reduction of $\geq 50 \%$ with SPEP during the study (OR 24.09, $p=$ $0.004)$, but not with ISS (OR $=1.31, p=0.813)$. The sensitivity and specificity of our prediction model were respectively 81 and $89 \%$.

Using sFLCs, patients with unstable response had a significantly worse 1-year PFS compared to patients with stable response $(36 \%$ vs. $57 \%, p=0.023$, Fig. $1 \mathrm{~B}$ ) regardless of ilg results at end of cycle 3 . In multivariate analysis, ISS was stratified, and only 
A $\begin{aligned} & \text { SFLC reduction } \\ & \text { of paraprotein }\end{aligned}-<90 \% \quad->>90 \%$
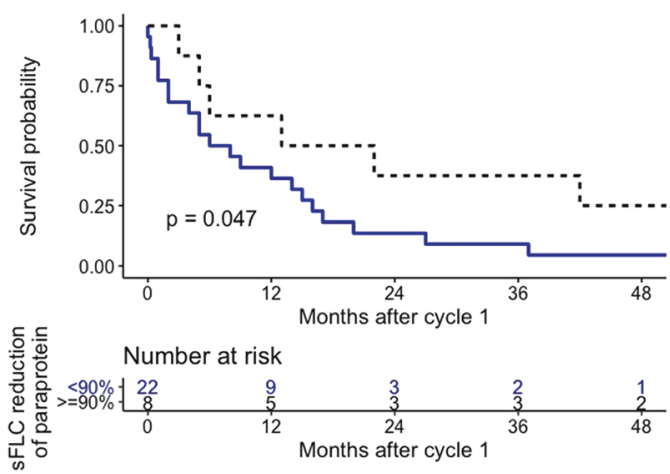

B
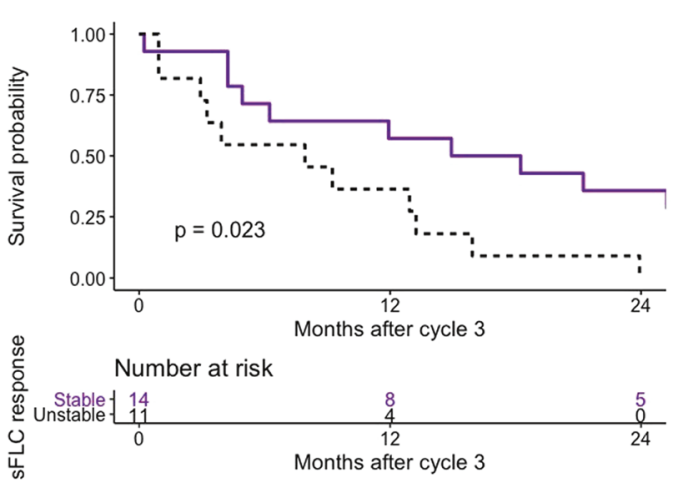

Fig. 1 Progression-free survival according to sFLC response. A Shown is the progression-free survival (PFS) according to sFLC reduction of paraprotein after cycle 1 and B progression-free survival (PFS) by the stability of early sFLC response.

unstable response was predictive of progression (HR 3.42, $p=$ 0.037). SPEP and HLC paraprotein fluctuations were not observed.

This study is the first to prospectively study and compare early treatment responses by SPEP, sFLC, and HLC on the outcome of ilg MM. sFLCs are a good real time indicator of tumor burden, including in ilg MM. As reported by Mead [7] and Pratt [8], we confirm that the decrease in paraprotein measured by SPEP and SFLCs occurred early after treatment initiation, with the latter showing a more rapid reduction due to a shorter serum half-life of sFLCs. We also confirmed previous observations made by Dispenzieri et al. who reported that SFLCs allow detection of early response among ilg MM and correlate with a favorable prognosis [9]. In our study, we evaluated patients receiving modern treatments, including bortezomib and IMiDs with several paraprotein evaluations. Our data indicate that early assessment of sFLCs as early as cycle 1 has a significant impact on prognosis. Our results are also consistent with Holzhey et al. who demonstrated that rapid sFLC decrease on day 8 is an early prognostic marker for newly diagnosed patients undergoing bortezomib-based treatments [10].

Hansen et al. demonstrated that a $>80 \%$ reduction in sFLCs 21 days after initiation of treatment was strongly associated with the achievement of $\geq$ VGPR [11]. Their results are consistent with the fact that obtaining a normal sFLC ratio confers a more favorable prognosis, irrespective of the depth of response [12]. Similarly, we have shown that patients failing to reduce sFLCs by at least $50 \%$ by the end of cycle 1 had significantly shorter PFS, independently of ISS and type of treatment received.

Few studies have reported the outcome of patients experiencing unstable sFLCs $[11,13,14]$. These fluctuations likely reflect a temporary inhibition of tumor synthesis rather than tumor killing [13] and could be an early indication of tumor resistance, not immediately evident in serial measurements of SPEP due to a longer half-life of ilgs. In our study, patients with an unstable response using SFLC assay had a significantly worse prognosis.

In contrast to sFLCs, there is little published data on the use of HLC monitoring after initiation of treatment. The response was detected at the same time by HLC and SPEP in most of our patients [15], which is unsurprising considering the serum half-life of heavy chains. Response by sFLC was evident much earlier. Of interest, Bradwell et al. [5] reported a case of early progression in a patient with IgG MM and increasing HLC ratio during the first chemotherapy cycle while the M-protein was decreasing. A rapid paraprotein increase was observed after cycle 2 with both assays. One of our patients behaved similarly, concomitantly with an unstable sFLC levels.

This single-institution, prospective study has limitations, including the fact that a small sample size limits detection of differences in PFS between each different groups with a heterogeneous population. Lack of bone marrow aspirate/biopsy and urinary
Bence Jones evaluations also limited correlations with IMWG response criteria. Nonetheless, our results show that early reduction in $\mathrm{sFLC}$ is clearly predictive of a better clinical outcome and the assay could potentially be used to optimize therapy.

In conclusion, patients with ilg MM presenting early sFLC improvement at the end of cycle 1 have a better prognosis, even without apparent response by SPEP. Moreover, patients with unstable sFLC response have significantly shorter PFS. Although our observations need to be confirmed in larger cohorts of patients, we believe that early sFLC measurements are predictive of clinical outcomes and could be used to personalize therapy.

\section{Jean-Sébastien Claveau $\mathbb{D}^{1 凶}$, Sophie Savary Bélanger ${ }^{1}$, Imran Ahmad ${ }^{1}$, Jean-Sébastien Delisle ${ }^{1}$, Vincent De Guire ${ }^{2}$, Jean Roy ${ }^{1}$ and Richard LeBlanc ${ }^{1}$ \\ ${ }^{1}$ Division of Hematology, Oncology and Transplantation, Department of Medicine, Maisonneuve-Rosemont Hospital, Université de Montréal, Montreal, QC, Canada. ${ }^{2}$ Department of Biochemistry, Maisonneuve-Rosemont Hospital, Université de Montréal, Montreal, QC, Canada. \\ 凶email: jean-sebastien.claveau@umontreal.ca}

\section{REFERENCES}

1. Kyle RA, Gertz MA, Witzig TE, Lust JA, Lacy MQ, Dispenzieri A, et al. Review of 1027 patients with newly diagnosed multiple myeloma. Mayo Clin Proc. 2003;78:21-33.

2. Dispenzieri A, Kyle R, Merlini G, Miguel JS, Ludwig H, Hajek R, et al. International Myeloma Working Group guidelines for serum-free light chain analysis in multiple myeloma and related disorders. Leukemia 2009;23:215-24.

3. Snozek CL, Katzmann JA, Kyle RA, Dispenzieri A, Larson DR, Therneau TM, et al. Prognostic value of the serum free light chain ratio in newly diagnosed myeloma: proposed incorporation into the international staging system. Leukemia 2008;22:1933-7.

4. Kumar S, Paiva B, Anderson KC, Durie B, Landgren O, Moreau P, et al. International Myeloma Working Group consensus criteria for response and minimal residual disease assessment in multiple myeloma. Lancet Oncol. 2016;17:e328-e46.

5. Bradwell AR, Harding SJ, Fourrier NJ, Wallis GL, Drayson MT, Carr-Smith HD, et al. Assessment of monoclonal gammopathies by nephelometric measurement of individual immunoglobulin kappa/lambda ratios. Clin Chem. 2009;55:1646-55.

6. Durie BG, Harousseau JL, Miguel JS, Blade J, Barlogie B, Anderson K, et al. International uniform response criteria for multiple myeloma. Leukemia 2006;20:1467-73.

7. Mead GP, Carr-Smith HD, Drayson MT, Morgan GJ, Child JA, Bradwell AR. Serum free light chains for monitoring multiple myeloma. $\mathrm{Br} J$ Haematol. 2004;126:348-54.

8. Pratt G, Mead GP, Godfrey KR, Hu Y, Evans ND, Chappell MJ, et al. The tumor kinetics of multiple myeloma following autologous stem cell transplantation as assessed by measuring serum-free light chains. Leuk Lymphoma. 2006;47:21-8.

9. Dispenzieri A, Zhang L, Katzmann JA, Snyder M, Blood E, Degoey R, et al. Appraisal of immunoglobulin free light chain as a marker of response. Blood 2008;111:4908-15. 
10. Holzhey T, P"nisch W, Wang SY, Holzvogt M, Holzvogt B, Andrea M. et al. Prognostic impact of rapid reduction of involved free light chains in multiple myeloma patients under first-line treatment with Bendamustine, Prednisone, and Bortezomib (BPV). J Cancer Res Clin Oncol. 2021;147:2349-2359.

11. Hansen CT, Pedersen PT, Nielsen LC, Abildgaard N. Evaluation of the serum free light chain ( $\mathrm{F} F \mathrm{LC}$ ) analysis in prediction of response in symptomatic multiple myeloma patients: rapid profound reduction in involved FLC predicts achievement of VGPR. Eur J Haematol. 2014;93:407-13.

12. Alhaj Moustafa M, Rajkumar SV, Dispenzieri A, Gertz MA, Lacy MQ, Buadi FK, et al. Utility of serum free light chain measurements in multiple myeloma patients not achieving complete response to therapy. Leukemia 2015;29:2033-8.

13. Fuchida $S$, Okano A, Hatsuse $M$, Murakami $S$, Haruyama $H$, Itoh $S$, et al. Seria measurement of free light chain detects poor response to therapy early in three patients with multiple myeloma who have measurable M-proteins. Int J Hematol. 2012;96:664-8

14. Das M, Mead GP, Sreekanth V, Anderson J, Howe T, Cavet J. et al. Serum free light chain (SFLC) concentration kinetics in patients receiving bortezomib: Temporary inhibition of protein synthesis and early biomarker for disease response. Blood. 2005; 106:5094

15. Michallet $M$, Chapuis-Cellier C, Dejoie T, Lombard C, Caillon $H$, Sobh $M$, et al. Heavy+light chain monitoring correlates with clinical outcome in multiple myeloma patients. Leukemia 2018;32:376-82.

\section{AUTHOR CONTRIBUTIONS}

JSC, SSB, and RL designed the study and wrote the manuscript. JSC, RL, and IA analyzed the data. JSC performed the statistical analysis. IA, JSD, and VDG provided suggestions and revisions. JR and RL supervised the analysis. All authors read and approved the final manuscript.

\section{COMPETING INTERESTS}

The authors declare no competing interests.

\section{ADDITIONAL INFORMATION}

Correspondence and requests for materials should be addressed to Jean-Sébastien Claveau.

Reprints and permission information is available at http://www.nature.com/ reprints

Publisher's note Springer Nature remains neutral with regard to jurisdictional claims in published maps and institutional affiliations.

Open Access This article is licensed under a Creative Commons Attribution 4.0 International License, which permits use, sharing, adaptation, distribution and reproduction in any medium or format, as long as you give appropriate credit to the original author(s) and the source, provide a link to the Creative Commons license, and indicate if changes were made. The images or other third party material in this article are included in the article's Creative Commons license, unless indicated otherwise in a credit line to the material. If material is not included in the article's Creative Commons license and your intended use is not permitted by statutory regulation or exceeds the permitted use, you will need to obtain permission directly from the copyright holder. To view a copy of this license, visit http://creativecommons. org/licenses/by/4.0/.

(c) The Author(s) 2021 